\title{
A comparison of three-dimensional rotation formalisms for least-squares and Bayesian inverse kinematics
}

\author{
Ben Serrien $^{\mathrm{a}}$, Klevis Aliaj ${ }^{\mathrm{b}, \mathrm{c}}$, Todd Pataky ${ }^{\mathrm{d}}$ \\ ${ }^{a}$ Experimental Anatomy Research Group, Vrije Universiteit Brussel, Brussels, Belgium \\ ${ }^{b}$ Department of Biomedical Engineering, University of Utah, Salt Lake City, Utah, USA \\ ${ }^{c}$ Department of Orthopaedics, University of Utah, Salt Lake City, Utah, USA \\ ${ }^{d}$ Department of Human Health Sciences, Kyoto University, Kyoto, Japan
}

\begin{abstract}
Marker-based inverse kinematics (IK) is prone to errors arising from measurement noise and soft-tissue artefacts. Various least-squares and Bayesian methods can be applied to limit the estimation error to a minimum. Recently proposed methods like Bayesian IK come at an increased computational cost however. In this technical paper, we present an overview of eight different least squares or Bayesian IK methods, including their accuracy and computational load for IK problems involving a single rigid body and three rotational degrees-of-freedom, whose attitude is estimated from four noisy marker positions. The results indicate that Non-Linear Least Squares, Variational Bayesian and full Bayesian IK are superior to Singular Value Decomposition in terms of accuracy, with approximately a two-fold error reduction. However, only Non-Linear Least Squares and Variational Bayesian IK are computationally efficient enough to scale towards practical use in biomechanical applications, with computational durations of 1-10 ms; fully Bayesian procedures required approximately $30 \mathrm{~s}$ for single rotation calculations. All Python code and supplementary material can be found in this paper's GitHub repository: https://github.com/benserrien/pybik.
\end{abstract}

Keywords: Biomechanics, 3D kinematics, Bayesian inference, Variational

Bayes, quaternion, $S O(3)$ 


\section{Introduction}

Marker based inverse kinematics (IK) uses the coordinates of a set of markers to infer the underlying set of motion parameters $\boldsymbol{\theta}$. In biomechanical analyses, the task of IK is to infer the joint angles between two neighboring segments or to infer the rotation of a joint between two successive time points. In 3D kinematics involving joints with three rotational degrees-offreedom (DOF), the parameter of interest belong to the Special Orthogonal group: $\boldsymbol{\theta} \in S O(3)$. Various representations of the $S O(3)$ group exist, of which the most commonly known are the rotation matrix, the rotation vector (also known as helical axis or axis-angle), Euler/Cardan angles and unit quaternions (Zatsiorsky, 1998; Bottema and Roth, 1979).

The presence of measurement noise and soft-tissue artefacts complicates IK procedures because not the true, but noisy coordinates are observed. Classically, several least-squares (LS) methods have been used to solve this task, but recently Bayesian methods have been suggested. Rather than minimizing the sum of squared errors of marker positions given the motion parameters, Bayesian IK (BIK) tries to maximize the posterior probability of the parameters given the observed marker positions and certain prior beliefs on the parameter values. Pataky et al. (2019) demonstrated a major advantage of BIK in terms of accuracy for a series of planar rigid-body IK problems. In Serrien et al. (2020), an attempt was made to extend BIK to 2D IK problems including soft-tissue artefacts and to 3D problems. These extensions proved only more accurate than LS-IK when the measurement noise was much larger ( $\sim 20 \mathrm{~mm}$ ) than can be expected for modern motion capture equipment.

However, Serrien et al. (2020) tested a 6-DOF model using only the Finite 
Helical Axis representation. In order to clarify BIK's computational limitations, and to ultimately improve BIK, here we consider simpler IK problems involving only a single rigid body and 3 rotational DOFs. In order for this research line to mature towards practical applications, computation time is another parameter to consider. Full Bayesian methods are computationally very intensive, making them unattractive for large-scale IK problems or use in biomechanical software. Approximation methods, like Variational Bayes and non-linear least squares (Chappell et al., 2009; Solà, 2017), are appealing in their potential to provide the same level of accuracy as full Bayesian methods while maintaining computational efficiency.

The aims of this technical paper are therefore (1) to compare different $S O(3)$ representations in terms of their performance in three-dimensional BIK and (2) to examine if approximation methods including Variational Bayes and non-linear least squares can be used to balance the requirements of accuracy and computational burden.

\section{Methods}

\subsection{D Forward and Inverse Kinematics}

The forward kinematics model we considered is an active rotation of a rigid body from the initial (0) to final (1) positions under influence of $\boldsymbol{\theta}$ : $\boldsymbol{r}_{i 1}=\boldsymbol{R}(\boldsymbol{\theta}) \boldsymbol{r}_{i 0}$, where $\boldsymbol{r}_{i}$ is a position vector representing the $i$ th point on the body which is considered to be rigidly fixed to the body. The rotation matrix $\boldsymbol{R}$ is constructed according to the specific representation of $\boldsymbol{\theta}$. In inverse kinematics, the objective is to estimate $\boldsymbol{\theta}$ given the observed, noisy marker coordinates. 
We evaluated two LS-IK methods in terms of their accuracy and computational speed: (1) singular value decomposition (SVD) (Söderkvist and Wedin, 1993) and (2) quaternion-based LS-IK (lsQUAT) (Horn, 1987). SVD is the most often used IK method and will therefore serve as the reference. For BIK, we evaluated four full Bayesian models according to the different representations: (1) rotation vector model (BIKrotvec), (2) intrinsic Euler YXY sequence (BIKeuler), (3) intrinsic Cardan ZXY sequence (BIKcardan) and (4) quaternion (BIKquat). The Euler/Cardan sequences were chosen because they are the ISB-recommended sequences for most joint coordinate systems (Wu et al., 2002, 2005). These BIK models were fitted to the noisy marker data using Markov Chain Monte Carlo procedures using the PyMC 2.3.8 package (Patil et al., 2010). Additionally, in order to explore more computationally efficient methods, the quaternion representation was modeled using the Variational Bayesian algorithm presented in Chappell et al. (2009). The Variational Bayes algorithm converges to the non-linear least squares (NLLS) solution in the case of non-informative priors, therefore a NLLS algorithm (Chappell et al., 2009; Solà, 2017) was also implemented. In Appendix A, we present the mathematical and statistical details of the different methods. Python implementations of each method are available in the package PyBIK in the project's public repository, along with a notebook demonstrating the use of the package (Appendix B), and a simulation script that replicates this study's results.

\subsection{Simulation experiment and error metric}

In order to test the accuracy of the different methods, simulation experiments are necessary because with in-vivo measurements the true rotation 
is unknown. All simulations were conducted in Python 3.7.7 (Van Rossum, 2020) using the Spyder IDE (Anaconda, 2020).

In the simulations we used $n=4$ markers and assume that the noise is Gaussian, isotropic and uncorrelated: $\tilde{\boldsymbol{r}}_{i}=\boldsymbol{r}_{i}+\boldsymbol{\epsilon}_{i}$ with $\epsilon_{i j} \stackrel{i . i . d .}{\sim} \mathcal{N}\left(0, \sigma^{2}\right)$ $(i=1, \ldots, n, j=x, y, z)$. For the standard deviation of the noise, we used the following levels which cover the range of expected noise levels for modern-day opto-electronic motion capturing: $\sigma=0.2,1.0,2.0 \mathrm{~mm}$ (Chen et al., 1994; Windolf et al., 2008). For each noise level, we simulated 1000 replicates of known random 3D rotations and performed IK using the different LS and Bayesian methods. Within these replicates, we performed uniform subsampling of the rotation magnitude (range: $0-\pi$ ), size of the marker plate (range: $75^{2}-150^{2} \mathrm{~mm}^{2}$ ) and distance of the markerplate to the rotation center (range: $100-400 \mathrm{~mm}$ ).

The estimation error for each IK method is calculated according to Equations (1) and (2) using the metric described in Huynh (2009) (metric 6 from that paper). First, the difference $\left(q^{\prime}\right)$ between the estimated $(\hat{q})$ and true $\left(q_{\text {true }}\right)$ orientation - each represented via unit quaternions - is computed:

$$
q^{\prime}=\hat{q} \cdot q_{\text {true }}^{-1}
$$

92 Then, the magnitude of the error is computed:

$$
\left|q^{\prime}\right|=2 \cdot \arctan \left(\frac{\sqrt{q_{x}^{\prime 2}+q_{y}^{\prime 2}+q_{z}^{\prime 2}}}{q_{w}^{\prime}}\right)
$$

3 The value of $\left|q^{\prime}\right|$ can be interpreted as the smallest angle between the true and estimated rotation. Appendix B explains why a simple subtraction of Euler/Cardan angles, rotation vectors, or quaternions are not proper error 
metrics. Descriptive statistics of the errors are calculated and all methods are compared to the standard SVD method.

\section{Results}

Table 1 and Figure 1 present descriptive statistics of the accuracy and computation time for each method. For all values of the measurement noise, we see that SVD and lsQUAT resulted in the same estimation (numerical differences occur after approximately 13 decimal places). The NLLS and (V)BIK methods were significantly more accurate than SVD for all noise levels (approximately $90 \%$ of simulations resulted in more accurate estimates). With increasing noise levels, the advantage of these methods over SVD increases; at $\sigma=0.2 \mathrm{~mm}$ the mean difference between SVD and the other methods was $0.07^{\circ}$ and rose to $0.36^{\circ}$ and $0.72^{\circ}$ for $\sigma=1.0$ and $2.0 \mathrm{~mm}$ respectively. Among the more accurate methods, only small differences were seen in the accuracy at the lowest noise level, where the (V)BIK methods had a small advantage over NLLS. In Appendix C, the errors are plotted against the rotation magnitude, distance of the marker plate to the origin and size of the marker plate. Only the latter variable had a small effect on the error (higher average error with smaller marker plates). This effect was smaller for NLLS and (V)BIK, demonstrating that they are less sensitive to the scale of the model.

In terms of computation time, lsQUAT and SVD were clearly the fastest methods, completing in under $1 \mathrm{~ms}$, followed by NLLS and VBIK, which completed in approximately 2 and $10 \mathrm{~ms}$, respectively. The BIK methods using MCMC were orders-of-magnitude-slower, completing in an average du- 
Table 1: Median IK errors, percentage of simulations with better accuracy than SVD and computation times for different Bayesian ans LS methods. More descriptive statistics can be found in Appendix C.

\begin{tabular}{lccccccc}
\hline & \multicolumn{2}{c}{$\sigma=0.2 \mathrm{~mm}$} & \multicolumn{2}{c}{$\sigma=1.0 \mathrm{~mm}$} & \multicolumn{2}{c}{$\sigma=2.0 \mathrm{~mm}$} & computation time (s) \\
& median $\left(^{\circ}\right)$ & $\%$ & $\operatorname{median}\left(^{\circ}\right)$ & $\%$ & $\operatorname{median}\left({ }^{\circ}\right)$ & $\%$ & mean \\
\cline { 2 - 8 } SVD & 0.139 & - & 0.731 & - & 1.440 & - & $3.49 \mathrm{E}-04$ \\
lsQUAT & 0.139 & 53 & 0.731 & 48 & 1.440 & 49 & $2.67 \mathrm{E}-04$ \\
NLLS & 0.085 & 57 & 0.364 & 89 & 0.699 & 92 & $1.78 \mathrm{E}-03$ \\
VBIK & 0.072 & 91 & 0.365 & 91 & 0.699 & 92 & $1.03 \mathrm{E}-02$ \\
BIKeuler & 0.077 & 89 & 0.366 & 90 & 0.701 & 91 & $5.28 \mathrm{E}+01$ \\
BIKcardan & 0.074 & 90 & 0.364 & 91 & 0.700 & 92 & $5.26 \mathrm{E}+01$ \\
BIKrotvec & 0.077 & 89 & 0.364 & 90 & 0.689 & 92 & $3.22 \mathrm{E}+01$ \\
BIKquat & 0.073 & 90 & 0.367 & 91 & 0.696 & 91 & $3.06 \mathrm{E}+01$ \\
\hline
\end{tabular}
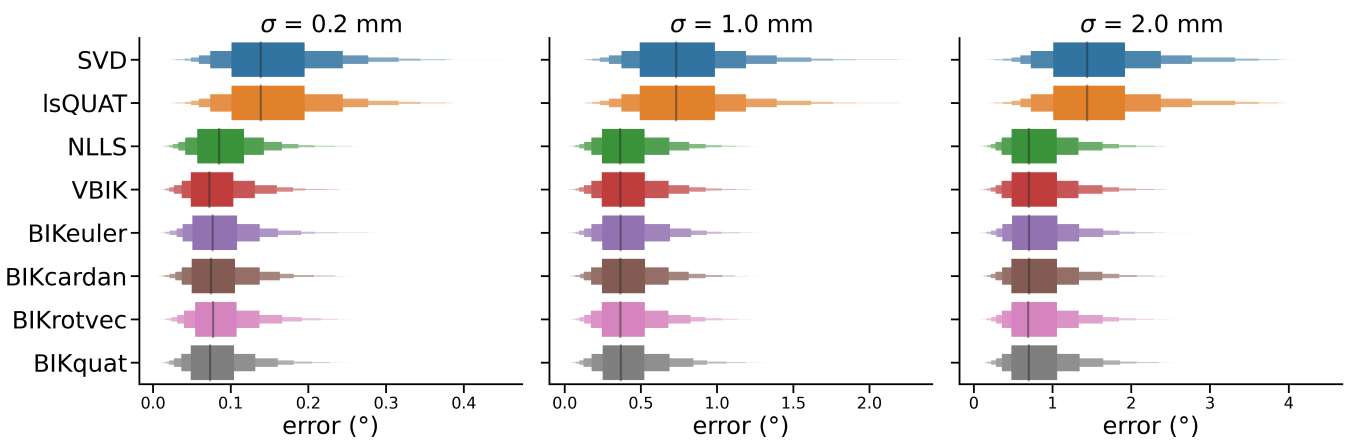

Figure 1: Distribution of inverse kinematics estimation errors as a function of method and noise level. The black line and most central box represent the median and first/third quantiles, other percentiles are shown as more narrow boxes. Note the different scales of the $x$-axes in the subplots. 


\section{Discussion}

The results of this simulation experiment demonstrate a clear advantage of NLLS and (V)BIK over classical LS-IK. In approximately $90 \%$ of the simulations, these methods more accurately estimated the rotation than SVD. While full BIK models using MCMC are computationally very intensive and therefore unattractive for practical use, an approximation like VBIK may be more practical, because our results suggest that VBIK is much faster without a loss in accuracy. While NLLS also gave more accurate estimates than SVD, it has less flexibility than VBIK for further research and development.

The difference between SVD (estimating the rotation matrix) and lsQUAT (estimating the quaternion) was negligible. Among the full BIK models, only small differences were observed between the $S O(3)$ representations. The primary difference between the representations lies in computational efficiency; the quaternion-based methods were significantly faster, both within BIK and LS-IK. The VBIK approach also made use of quaternions because this was the only representation that could be modelled using conjugate priors (Appendix A). Other Bayesian IK algorithms like Sequential Monte Carlo (Courty and Arnaud, 2008) also employ the unit quaternion representation for their computational and numerical efficiency. While the four quaternion components cannot be directly interpreted in terms of biomechanically relevance, they can be readily transformed into more meaningful quantities like Euler angles.

The advantage of Bayesian over least-squares methods decreased with smaller noise levels. With continuing technological improvements in optoelectronic motion capturing, the advantage of (V)BIK may therefore diminish. Inertial measurement units are generally much noisier and may ben- 
efit from (V)BIK more. Soft-tissue artefacts (STA) (Bonnet et al., 2017; Camomilla et al., 2017) are another area where (V)BIK may prove advantageous. In Serrien et al. (2020), STAs were modelled using an explicit forward model, but results of BIK were only more accurate than SVD at noise levels far above realistic ones (i.e., up to a noise standard deviation of $20 \mathrm{~mm}$ ). When using marker plates, an explicit kinematic STA-model can be devised to describe and thus account for STAs because all markers are affected equally. However, STAs are primarily problematic when markers are attached to the skin. When applying (V)BIK in such a setting, an explicit forward kinematic STA-model is hard to validate. It can nevertheless be statistically modelled using a dynamic heteroscedastic noise model, that uses marker-specific and time-varying noise levels (the STA depends on the local skin properties and the dynamics of the movement).

Another potential advantage of VBIK lies in kinematic time series data. The present simulations were limited to (time-independent) discrete rotations, but in biomechanics, the objective is usually to conduct IK on dynamically rotating body segments, and to calculate joint angle trajectories. When the motion capture sampling frequency is sufficiently high, the movement is expected to be smooth and thus the posterior distribution at time point $t$ could be used to construct an informed prior for time point $t+1$. With VBIK we now have a new method that combines accuracy and computational speed that could be tested on time series data.

In the current simulation experiment, an important limitation is that we discussed only rotations for which the rotation vector passed through the origin, i.e. we assumed that the joint center was known with absolute 
precision. In biomechanics, joint center estimation is prone to errors as well (Kainz et al., 2015), so the (V)BIK models should be expanded to incorporate this error. This requires estimation of an additional 3-dimensional parameter (location of the joint center) from the same amount of data. This parameter may be strongly correlated with the rotation itself and could be problematic for model fitting, as suggested in Serrien et al. (2020). Another limitation is the use of simulations, which require noise models that may or may not be reflective of true measurement noise. Empirical validation studies using for instance using a robotic arm with controlled (known) rotations should be conducted to verify the current results.

Conflict of interest. All authors declare that they have no conflicts of interest regarding the contents of this paper.

\section{References}

Anaconda, . Anaconda software distribution, 2020. URL https://docs . anaconda.com/.

Bonnet, V., Dumas, R., Cappozzo, A., Joukov, V., Daune, G., Kulic, D., Fraisse, P., Andary, S., and Venture, G. A constrained extended kalman filter for the optimal estimate of kinematics and kinetics of a sagittal symmetric exercise. Journal of Biomechanics, 62:140-147, 2017.

Bottema, O. and Roth, B. Theoretical Kinematics. North-Holland Publishing Company, Dover Publications, New York, 1979. 
Camomilla, V., Dumas, R., and Cappozzo, A. Human movement analysis: The soft tissue artefact issue. Journal of Biomechanics, 62:1-4, 2017.

Chappell, M. A., Groves, A. R., Whitcher, B., and Woolrich, M. W. Variational Bayesian inference for a nonlinear forward model. IEEE Transactions on Signal Processing, 57(1):223-236, 2009.

Chen, L., Armstron, C.W., and Raftopoulos, D.D. An investigation on the accuracy ofthree-dimensional space reconstruction using the direct linear transformation technique. Journal of Biomechanics, 27:493-500, 1994.

Courty, N. and Arnaud, E. Inverse kinematics using sequential monte carlo methods. In Perales, F. J. and Fisher, R.B., editors, Articulated Motion and Deformable Objects, pages 1-10, Berlin, Heidelberg, 2008. Springer.

Horn, B.K.P. Closed-form solution of absolute orientation using unit quaternions. Journal of the Optical Society of America A, 4(4):629-642, 1987.

Huynh, D. Q. Metrics for 3d rotations: Comparison and analysis. Journal of Mathematical Imaging and Vision, 35(2):155-164, 2009.

Kainz, H., Carty, C.P., Modenese, L., Boyd, R.N., and Lloyd, D.G. Estimation of the hip joint centre in human motion analysis: A systematic review. Clinical Biomechanics, 30(4):319-329, 2015.

Pataky, T. C.., Vanrenterghem, J., and Robinson, M. A. Bayesian inverse kinematics vs. least-squares inverse kinematics in estimates of planar postures and rotations in the absence of soft tissue artifact. Journal of Biomechanics, 82:324-329, 2019. 
Patil, A., Huard, D., and Fonnesbeck, C. J. Pymc: Bayesian stochastic modelling in python. Journal of Statistical Software, 35(4), 2010.

Serrien, B., Pataky, T., Baeyens, J.P., and Cattrysse, E. Bayesian vs. leastsquares inverse kinematics: simulation experiments with models of 3D rigid body motion and $2 \mathrm{D}$ models including soft-tissue artefacts. Journal of Biomechanics, 109:109902, 2020.

Söderkvist, I. and Wedin, P. A. Determining the movements of the skeleton using well-configured markers. Journal of Biomechanics, 26:1473-1477, 1993.

Solà, J. Quaternion kinematics for the error-state kalman filter, 2017.

Van Rossum, G. The python library reference release 3.7.7, 2020. URL https://docs. python .org/3/library/.

Windolf, M., Gotzen, N., and Morlock, M. Systematic accuracy and precision analysis ofvideo motion capturing systems - exemplified on the vicon-460 system. Journal of Biomechanics, 41:2776-2780, 2008.

Wu, G., Siegler, S., P., Allard, and et al., . ISB recommendation on definitions of joint coordinate system of various joints for the reporting of human joint motion - part I: ankle, hip, and spine. Journal of Biomechanics, 35(4):543$548,2002$.

Wu, G., van der Helm, F.C., Veeger, H.E., and et al., . ISB recommendation on definitions of joint coordinate systems of various joints for the reporting of human joint motion - part II: shoulder, elbow, wrist and hand. Journal of Biomechanics, 38(5):981-992, 2005. 
${ }_{238}$ Zatsiorsky, V. Kinematics of Human Motion. Human Kinetics, Leeds, 1998. 


\title{
A comparison of three-dimensional rotation formalisms for least-squares and Bayesian inverse kinematics Appendix A
}

\author{
Ben Serrien, Klevis Aliaj, Todd Pataky
}

Mathematical and statistical details of the inverse kinematic methods that are omitted in the main paper are described here. This appendix is not meant to be a review or full derivation of these methods, we refer to the reference list for that purpose. It is meant to present the details of the different methods in a single notational framework and to serve as an explanation of the code used in the simulations. In Appendix B, a python notebook explains the use of the package.

\section{$13 \mathrm{D}$ rigid body kinematics and the $S O(3)$ group}

In the main paper we describe the accuracy of inverse kinematics methods for rigid bodies with 3 rotational degrees of freedom. That is, we assume that a set of $n$ markers attached to a rigid body are displaced in 3D Euclidean space through a rotation of the body around an axis passing through the origin of a fixed reference frame. The coordinates of the markers are described by 3 -vectors $\boldsymbol{r}_{i}(i=1, \ldots, n)$. In the simulations we always used $n=4$, which is a number used in many biomechanical models. The forward kinematics equation describing this rotation between an initial (0) and final (1) position is the following:

$$
\boldsymbol{r}_{i 1}=\boldsymbol{R}(\boldsymbol{\theta}) \boldsymbol{r}_{i 0}
$$

with $\boldsymbol{R}(\boldsymbol{\theta}) \in \mathbb{R}^{3 \times 3}$ a rotation matrix. The objective of inverse kinematics (IK) is to find $\boldsymbol{\theta}$ based on the $3 \mathrm{D}$ coordinates. In reality, not the true but noisy coordinates are observed (measured): $\tilde{\boldsymbol{r}}_{i}=\boldsymbol{r}_{i}+\boldsymbol{\epsilon}_{i}$ which complicates the task. In the main paper we assume that the measurement noise is zero-mean uncorrelated Gaussian isotropic noise:

$$
\boldsymbol{\epsilon}_{i} \stackrel{i . i . d .}{\sim} \mathcal{N}\left(\mathbf{0}, \sigma^{2} \boldsymbol{I}_{3}\right)
$$

or equivalently because we assume uncorrelated and isotropic noise:

$$
\epsilon_{i j} \stackrel{i . i . d .}{\sim} \mathcal{N}\left(0, \sigma^{2}\right) \quad(j=x, y, z)
$$

The rotation matrix is only one of the representations of a 3D rotation. In general, rotations in 3D Euclidean space form an algebraic group, called the Special Orthogonal group $S O(3)$ which can be represented in various ways. All rotations are denoted by $\boldsymbol{\theta} \in S O(3)$. The rotation matrix can be constructed based on the specific representation of $\boldsymbol{\theta}$ and consists of 9 numbers while only 3 degrees of freedom are present in this setting. More compact representations are the rotation vector, Euler/Cardan angles and unit quaternions (explained below). In the package, we used the Rotation class from the scipy.spatial.transform module which incorporates these representations, conversions between them and other useful functions.

\subsection{Rotation vector}

The rotation vector representation is also known as the axis-angle or helical axis representation. The rotation vector is a pseudo-vector whose unit vector $(\boldsymbol{u})$ describes the orientation of the axis around which the rigid body is rotating and whose magnitude $(\theta)$ is the angle over which the rotation occurs. This representation consists thus of 4 numbers plus a unit vector constraint: $\boldsymbol{\theta}=(\boldsymbol{u}, \theta)=\left(u_{x}, u_{y}, u_{z}, \theta\right)$. The rotation matrix takes the following form:

$$
\boldsymbol{R}(\boldsymbol{u}, \theta)=\left(\begin{array}{ccc}
\cos \theta+u_{x}^{2}(1-\cos \theta) & u_{x} u_{y}(1-\cos \theta)-u_{z} \sin \theta & u_{x} u_{z}(1-\cos \theta)+u_{y} \sin \theta \\
u_{y} u_{x}(1-\cos \theta)+u_{z} \sin \theta & \cos \theta+u_{y}^{2}(1-\cos \theta) & u_{y} u_{z}(1-\cos \theta)-u_{x} \sin \theta \\
u_{z} u_{x}(1-\cos \theta)-u_{y} \sin \theta & u_{z} u_{y}(1-\cos \theta)+u_{x} \sin \theta & \cos \theta+u_{z}^{2}(1-\cos \theta)
\end{array}\right)
$$




\subsection{Euler and Cardan angles}

Euler and Cardan angles describe 3D rotations as a sequence of three rotations around a specific set of axes (in contrast to the rotation vector which describes it as a rotation over an angle around 1 axis). This representation thus consists of three numbers: $\boldsymbol{\theta}=(\alpha, \beta, \gamma)$. The International Society of Biomechanics has a set of guidelines (Wu et al., 2002, 2005) that describe the recommended sequence for intrinsic Euler and Cardan angles for various joint coordinate systems. For most joints, the ZXY sequence is recommended, but the YXY sequence is used for the shoulder. The rotation matrices for these sequences are given below with $\alpha, \beta, \gamma$ the angles corresponding to the first, second and third rotations respectively:

$$
\begin{gathered}
\boldsymbol{R}_{Z X Y}(\alpha, \beta, \gamma)=\left(\begin{array}{ccc}
\cos \alpha \cos \gamma-\sin \alpha \sin \beta \sin \gamma & -\cos \beta \sin \alpha & \cos \alpha \sin \gamma+\cos \gamma \sin \alpha \sin \beta \\
\cos \gamma \sin \alpha+\cos \alpha \sin \beta \sin \gamma & \cos \alpha \cos \beta & \sin \alpha \sin \gamma-\cos \alpha \cos \gamma \sin \beta \\
-\cos \beta \sin \gamma & \sin \beta & \cos \beta \cos \gamma
\end{array}\right) \\
\boldsymbol{R}_{Y X Y}(\alpha, \beta, \gamma)=\left(\begin{array}{ccc}
\cos \alpha \cos \gamma-\cos \beta \sin \alpha \sin \gamma & \sin \alpha \sin \beta & \cos \alpha \sin \gamma+\cos \beta \cos \gamma \sin \alpha \\
\sin \beta \sin \gamma & \cos \beta & -\cos \gamma \sin \beta \\
-\cos \gamma \sin \alpha-\cos \alpha \cos \beta \sin \gamma & \cos \alpha \sin \beta & \cos \alpha \cos \beta \cos \gamma-\sin \alpha \sin \gamma
\end{array}\right)
\end{gathered}
$$

\subsection{Unit quaternions}

Quaternions $(q)$ are 4-dimensional numbers, consisting of a complex vector part $(\boldsymbol{q})$ and a real scalar part $\left(q_{w}\right)$ : $q=\boldsymbol{q}+q_{w}=q_{x} \boldsymbol{i}+q_{y} \boldsymbol{j}+q_{z} \boldsymbol{k}+q_{w}$. See for example Horn (1987) for a reference on quaternion algebra in the context of $3 \mathrm{D}$ rotations. Unit quaternions are quaternions whose norm equals 1 . The relation between unit quaternions and the rotation vector representation is the following: $q=\boldsymbol{u} \sin \frac{\theta}{2}+\cos \frac{\theta}{2}$; it is seen that for each $\boldsymbol{u}$ and $\theta$, this gives a unit quaternion. Each rotation can thus be represented by a single parameter consisting of 4 numbers: $\boldsymbol{\theta}=q$. Conventional scalars and 3-vectors can be represented as quaternions with a zero-vector part and a zero-scalar part respectively. A rotation of a 3 -vector to its new position can be written equivalently as premultiplying it with a rotation matrix or using the quaternion product as follows ${ }^{1}$ :

$$
\boldsymbol{r}_{i 1}=\boldsymbol{R}(q) \boldsymbol{r}_{i 0}=q \otimes \boldsymbol{r}_{i 0} \otimes q^{*}
$$

The rotation matrix in terms of the components of the unit quaternion takes the following form:

$$
\boldsymbol{R}(q)=\left(\begin{array}{ccc}
q_{w}^{2}+q_{x}^{2}-q_{y}^{2}-q_{z}^{2} & 2\left(q_{x} q_{y}-q_{w} q_{z}\right) & 2\left(q_{x} q_{z}+q_{w} q_{y}\right) \\
2\left(q_{x} q_{y}+q_{w} q_{z}\right) & q_{w}^{2}-q_{x}^{2}+q_{y}^{2}-q_{z}^{2} & 2\left(q_{y} q_{z}-q_{w} q_{x}\right) \\
2\left(q_{x} q_{z}-q_{w} q_{y}\right) & 2\left(q_{y} q_{z}+q_{w} q_{x}\right) & q_{w}^{2}-q_{x}^{2}-q_{y}^{2}+q_{z}^{2}
\end{array}\right)
$$

Unit quaternions are known to be numerically more stable and not to suffer from singularities or gimbal lock like the Euler/Cardan angles (Zatsiorsky, 1998; Bottema and Roth, 1979).

\section{Least-Squares Inverse Kinematics (LS-IK)}

In LS-IK, finding $\boldsymbol{\theta}$ is done by minimizing the sum of squared deviations between the observed noisy markers and the predicted marker coordinates:

$$
\hat{\boldsymbol{\theta}}_{\mathrm{LS}}=\underset{\boldsymbol{\theta}}{\operatorname{argmin}} \sum_{i=1}^{n}\left(\tilde{\boldsymbol{r}}_{i 1}-\boldsymbol{R}(\boldsymbol{\theta}) \tilde{\boldsymbol{r}}_{i 0}\right)^{2}
$$

\subsection{Singular Value Decomposition (SVD)}

Söderkwist and Wedin (1993) described a LS-IK solution using SVD. The method works as follows. Let the mean-centered marker coordinates be defined as $\boldsymbol{A}=\left[\tilde{\boldsymbol{r}}_{0,1}-\overline{\boldsymbol{r}}_{0}, \ldots, \tilde{\boldsymbol{r}}_{0, n}-\overline{\boldsymbol{r}}_{0}\right]$ and $\boldsymbol{B}=\left[\tilde{\boldsymbol{r}}_{1,1}-\overline{\boldsymbol{r}}_{1}, \ldots, \tilde{\boldsymbol{r}}_{1, n}-\overline{\boldsymbol{r}}_{1}\right]$, both $\in \mathbb{R}^{3 \times n}\left(\overline{\boldsymbol{r}}_{0 / 1}=\frac{1}{n} \sum_{i=1}^{n} \tilde{\boldsymbol{r}}_{0 / 1, i}\right)$ and define $\boldsymbol{C}=\boldsymbol{B} \cdot \boldsymbol{A}^{T}$. The $3 \times 3$ matrix $\boldsymbol{C}$ can be decomposed using SVD:

$$
\boldsymbol{C}=\boldsymbol{U} \cdot \boldsymbol{D} \cdot \boldsymbol{V}^{T}
$$

The SVD estimate for the rotation matrix can now be written as:

$$
\hat{\boldsymbol{R}}=\boldsymbol{U} \cdot \operatorname{diag}(1,1, \operatorname{det}(\boldsymbol{U} \cdot \boldsymbol{V})) \cdot \boldsymbol{V}
$$

\footnotetext{
${ }^{1}$ The conjugate of $q=\boldsymbol{q}+q_{w}$ is given by $q^{*}=-\boldsymbol{q}+q_{w}$
} 


\subsection{Quaternion based LS-IK (IsQUAT)}

A similar algorithm to SVD was proposed by Horn (1987) using quaternion algebra. The matrix $\boldsymbol{C}$ is defined in the same way as for SVD; this matrix contains all the information necessary for finding the LS solution to the rotation (Horn, 1987). Now define the $4 \times 4$ matrix $\boldsymbol{N}$ :

$$
\boldsymbol{N}=\left(\begin{array}{cc}
\operatorname{trace}(\boldsymbol{C}) & \Delta^{T} \\
\Delta & \boldsymbol{C}+\boldsymbol{C}^{T}-\operatorname{trace}(\boldsymbol{C}) \boldsymbol{I}_{3}
\end{array}\right)
$$

where $\Delta=\left(C_{2,3}-C_{3,2}, C_{3,1}-C_{1,3}, C_{1,2}-C_{2,1}\right)^{T}$. Horn (1987) shows that minimizing the sum of squared errors is done by the unit quaternion $\hat{q}$ which is the eigenvector of $\boldsymbol{N}$ corresponding to its most positive eigenvalue $\left(\lambda_{m}\right) ; \boldsymbol{N} \hat{q}=\lambda_{m} \hat{q}$.

\subsection{Non-Linear Least-Squares (NLLS) IK}

Because of the similarity to Variational BIK (infra), the NLLS method will be used with the unit quaternion representation. In NLLS, the forward model $\boldsymbol{f}$ is approximated by a local linearization using a first-order Taylor series.

$$
\begin{aligned}
\boldsymbol{r}_{i 1}=\boldsymbol{f}_{i}(q) & =q \otimes \boldsymbol{r}_{i 0} \otimes q^{*} \\
& \approx \boldsymbol{f}_{i}(m)+\boldsymbol{J}_{i}(q-m)
\end{aligned}
$$

The Jacobian of the forward model is given by the following (Solà, 2017) ${ }^{2}$ :

$$
\boldsymbol{J}_{i}=\frac{\partial \boldsymbol{f}_{i}}{\partial q}=2\left[\boldsymbol{q}^{T} \boldsymbol{r}_{i 0} \boldsymbol{I}_{3}+\boldsymbol{q} \boldsymbol{r}_{i 0}^{T}+\boldsymbol{r}_{i 0} \boldsymbol{q}^{T}-q_{w}\left[\boldsymbol{r}_{i 0}\right]_{\times} \mid q_{w} \boldsymbol{r}_{i 0}+\boldsymbol{q} \times \boldsymbol{r}_{i 0}\right] \in \mathbb{R}^{3 \times 4}
$$

The full Jacobian of all markers is thus a $3 n \times 4$ real matrix. Minimizing the sum of squared errors under this approximation leads to an iterative update equation of $m$. Using the Gauss-Newton algorithm, this is given by:

$$
m^{(t+1)}=m^{(t)}+\left(\boldsymbol{J}^{(t) T} \boldsymbol{J}^{(t)}\right)^{-1} \boldsymbol{J}^{(t) T} \boldsymbol{k}^{(t)}
$$

with $\boldsymbol{k}^{(t)}=\tilde{\boldsymbol{r}}_{1}-\boldsymbol{f}\left(m^{(t)}\right)$ (a vector of length $3 n$ ); the residual between the observed and predicted coordinates at iteration $t$ (Chappell et al., 2009). At each iteration, $m$ is properly normalized to a unit quaternion and the Jacobian is evaluated using the noisy coordinates. For starting the iteration at a good guess, we used the SVD estimate $\left(m^{(0)}=\hat{q}_{\mathrm{SVD}}\right)$. Convergence of the NLLS algorithm can be defined using various criteria. In the main paper we defined convergence if the change between two succesive iterations was smaller than $0.01^{\circ}$.

\section{Bayesian Inverse Kinematics (BIK)}

The major difference between LS-IK and BIK is that LS-IK is a purely mathematical optimization procedure, while BIK is a stochastic inference method (statistical optimization). Distributional assumptions are assigned to both the rotation ("parameter of interest"), the measurement noise ("nuisance parameter") and to the final noisy marker coordinates ("data") $)^{3}$. Initial noisy marker coordinates are treated as constants. In BIK, finding $\boldsymbol{\theta}$ is done by maximizing the posterior probability of $\boldsymbol{\theta}$ given the noisy coordinates and the prior information:

$$
\hat{\boldsymbol{\theta}}_{\mathrm{BIK}}=\underset{\boldsymbol{\theta}}{\operatorname{argmax}} p\left(\boldsymbol{\theta}, \tau \mid \tilde{\boldsymbol{r}}_{1}\right)
$$

The posterior distribution is obtained using Bayes' rule:

$$
p\left(\boldsymbol{\theta}, \tau \mid \tilde{\boldsymbol{r}}_{1}\right) \propto p\left(\tilde{\boldsymbol{r}}_{1} \mid \boldsymbol{\theta}, \tau\right) \cdot p(\boldsymbol{\theta}) \cdot p(\tau)
$$

with $p(\boldsymbol{\theta})$ and $p(\tau)$ the prior distributions on the rotation and precision and $p\left(\tilde{\boldsymbol{r}}_{1} \mid \boldsymbol{\theta}, \tau\right)$ the likelihood of the data. Bayes' rule is written without the proportionality constant because this integral is often not tractable. Numerical sampling methods like Markov Chain Monte Carlo (MCMC) can be used to draw samples from the full posterior. Given enough samples, the posterior mean can be approximated by the mean of these samples and can be used as point estimate $\hat{\boldsymbol{\theta}}_{\mathrm{BIK}}$. The various representations of $\boldsymbol{\theta}$ lead to different BIK models and are explained below.

\footnotetext{
${ }^{2}[.]_{\times}$is the skew-symmetric operator giving the $3 \times 3$ matrix of the vector product

${ }^{3}$ In the software package that we used (PyMC2), the normal distribution is not characterized by the variance $\sigma^{2}$ but by the precision: $\tau=\frac{1}{\sigma^{2}}$ (inverse of the variance).
} 


\subsection{Common parts of all MCMC BIK models}

Each BIK model is comprised of a forward model, a likelihood for the data and a (set of) prior(s). The forward model predicts the values of the final coordinates given the noisy initial positions and the current value $\boldsymbol{\theta}$ that was sampled in the chain. For the likelihood a normal distribution was used, centered at the predicted value from the forward model and with a precision $\tau$. For the prior on the measurement precision, we used a Gamma prior with two small constants, reflecting weak prior information on the true values. The constants were chosen in preliminary simulations that yielded good results (using a completely uninformative Gamma prior could be done by setting both constants to 0). The Gamma prior has a peak at zero, acting therefore as a shrinkage prior, directing the estimation of $\tau$ to zero (low precision). This resulted in better estimates because with only 4 markers (12 data points), the precision is hard to estimate accurately. The specific form of the prior on the rotation $p(\boldsymbol{\theta})$ is specified below for each of the different $S O(3)$ parameterizations. In each case, we used vague uninformative priors, indicating no a-priori knowledge of the rotation.

This model structure is summarized in the directed acyclic graph of Figure 1.

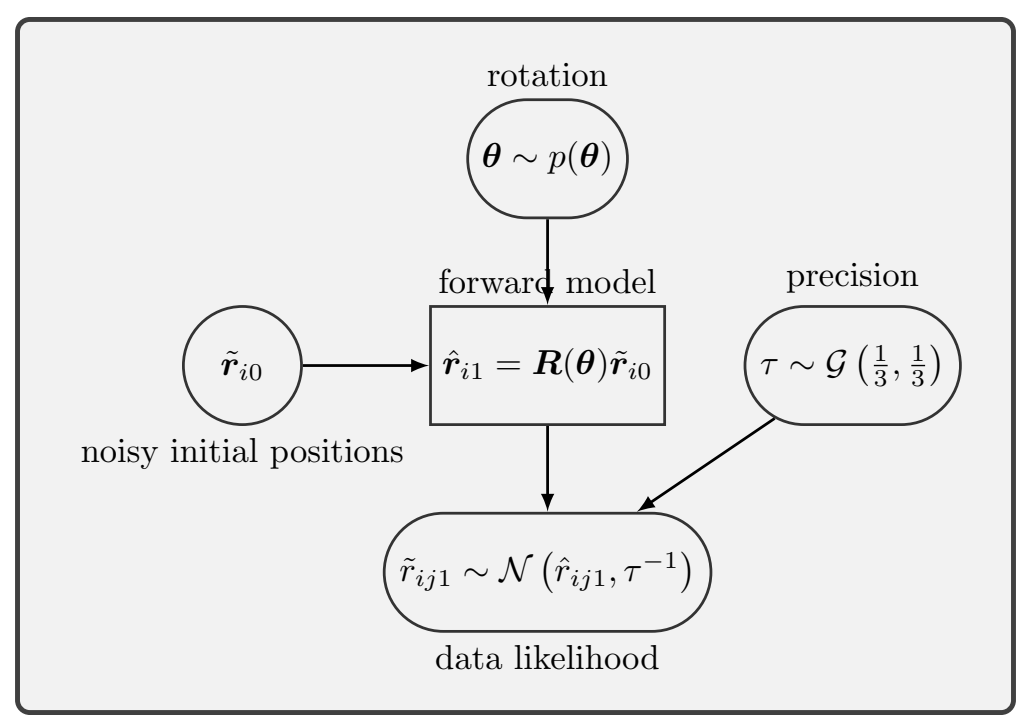

Figure 1: Directed acyclic graph of the generic BIK model. Stochastic variables are denoted by rounded rectangles (noisy final coordinates, rotation, precision), constants as circles (noisy initial positions) and deterministic random variables as rectangles (forward kinematics model).

To sample from the posterior distribution, Markov Chain Monte Carlo (MCMC) was used in the form of an adaptive Metropolis-Hastings algorithm. The parameters of this algorithm are given below; in preliminary simulation experiments we found these values to be stable and to provide good levels of accuracy.

- 150.000 iterations

- 50.000 burn-in

- thinning rate of 5

- updating the estimated covariance matrix every 10.000 iterations

- starting point for $\boldsymbol{\theta}$ : SVD estimate

- starting point for $\tau$ : true value (in laboratory settings, a good initial guess for $\tau$ can be obtained via dynamic trials with an object with known properties like a calibration wand)

\subsection{MCMC model using the rotation vector}

For the rotation vector formalism (axis-angle), we specified separate priors for the unit vector of the axis (multivariate standard normal) and the angle (uniform). A normalization was done before passing it to the forward model.

$$
\begin{aligned}
p\left(\boldsymbol{u}^{\prime}\right) & =\mathcal{N}\left(\mathbf{0}, \boldsymbol{I}_{3}\right) \quad \boldsymbol{u}=\boldsymbol{u}^{\prime} /\left|\boldsymbol{u}^{\prime}\right| \\
p(\theta) & =\mathcal{U}(0, \pi)
\end{aligned}
$$

In preliminary simulations, we tested also another model where we used uniform priors on the azimuthal and polar angles of the unit vector and transformed these to cartesian coordinates of. This model yielded very similar results and was therefore not used in the final simulation experiment. 


\subsection{MCMC model using Euler and Cardan angles}

Uniform priors were assigned to the Euler and Cardan angles according to their specific ranges ( $\alpha, \beta, \gamma$ correspond to the angles of the first, second and third rotations respectively):

$$
\begin{aligned}
p(\alpha) & =\mathcal{U}( \pm \pi) \\
p(\beta) & =\mathcal{U}(0, \pi) \quad \text { Euler } \\
p(\gamma) & =\mathcal{U}( \pm \pi) \\
p(\alpha) & =\mathcal{U}( \pm \pi) \\
p(\beta) & =\mathcal{U}\left( \pm \frac{\pi}{2}\right) \quad \text { Cardan } \\
p(\gamma) & =\mathcal{U}( \pm \pi)
\end{aligned}
$$

\subsection{MCMC model using unit quaternions}

A multivariate standard normal prior was given to the unnormalized quaternion. A normalization was done before passing it to the forward model.

$$
p\left(q^{\prime}\right)=\mathcal{N}\left(\mathbf{0}, \boldsymbol{I}_{4}\right) \quad q=q^{\prime} /\left|q^{\prime}\right|
$$

This normalization could be avoided by using a Von Mises-Fisher prior on the $S^{3}$ hypershpere . In preliminary simulation experiments we tested this option (using a concentration of zero, i.e. uniform) and found similar accuracy levels to the model using the normal prior. While the Von Mises-Fisher prior avoids the need for normalization, computation time was slightly higher because the sampling takes more time than with the normal prior.

\subsection{MCMC model using the rotation matrix}

In theory, the rotation matrix representation could also be used in MCMC-based BIK. The matrix-Von-MisesFisher distribution could be used as a prior distribution on $\boldsymbol{R}$ directly, but because this distribution is not proper, the posterior distribution is not proper either. In initial tests, the MCMC did not converge to a good solution using this representation (code in the package should not be used).

\section{Variational Bayesian Inverse Kinematics (VBIK)}

Whereas full Bayesian inference uses numerical sampling methods as MCMC to describe the posterior, Variational Bayesian inference uses an approximation to the true posterior that can be obtained using iterative optimization which is computationally much faster. Generally, this approximation comes at a cost of less accuracy (especially in the tails of the posterior). In the main paper we showed that this lower accuracy is not an issue for inverse kinematics (point estimation). The approximation works only for models where the likelihood comes from the exponential family of distributions and for conjugate priors. In BIK we always worked with a Normal likelihood, which is from the exponential family. A conjugate prior is a prior which, in combination with the likelihood gives a posterior that is of the same family. For a Normal likelihood with unknown mean and precision, the conjugate prior is a Normal and Gamma prior on the mean and precision respectively. This is akin to the BIK model using the unit quaternion representation. Other $S O(3)$ representations are impossible to model using conjugate Normal priors as the parameters of these representations are restricted to specific ranges and cannot be normalized (Euler/Cardan angles, angle of the rotation vector).

Chappell et al. (2009) described the following method for non-linear forward models using a Variational Bayes algorithm that can be directly applied to IK. The true posterior $p\left(q, \tau \mid \tilde{\boldsymbol{r}}_{1}\right)$ is approximated by a factorized posterior distribution $p^{\prime}\left(q, \tau \mid \tilde{\boldsymbol{r}}_{1}\right)=p^{\prime}\left(q \mid \tilde{\boldsymbol{r}}_{1}\right) \cdot p^{\prime}\left(\tau \mid \tilde{\boldsymbol{r}}_{1}\right)$ that can be obtained iteratively. The same linearization using a first-order Taylor series of the forward model as with NLLS is done to make the expectations tractable. The following priors are assigned to $q$ and $\tau$ :

$$
\begin{aligned}
& p^{\prime}(q)=\mathcal{N}\left(m_{0}, \boldsymbol{\Lambda}_{0}^{-1}\right) \quad\left(m_{0}=\hat{q}_{\mathrm{SVD}}, \boldsymbol{\Lambda}_{0}=1 \times 10^{-10} \boldsymbol{I}_{4}\right) \\
& p^{\prime}(\tau)=\mathcal{G}\left(s_{0}, c_{0}\right) \quad\left(s_{0}=c_{0}=1 / 3\right)
\end{aligned}
$$

leading to the following approximate posteriors:

$$
\begin{aligned}
& p^{\prime}\left(q \mid \tilde{\boldsymbol{r}}_{1}\right)=\mathcal{N}\left(m, \boldsymbol{\Lambda}^{-1}\right) \\
& p^{\prime}\left(\tau \mid \tilde{\boldsymbol{r}}_{1}\right)=\mathcal{G}(s, c)
\end{aligned}
$$


of which the parameters $m, \boldsymbol{\Lambda}, s, c$ can be derived via the following Expectation-Maximization (EM) scheme (Chappell et al., 2009):

$$
\begin{aligned}
c & =\frac{3 n}{2}+c_{0} \\
\boldsymbol{\Lambda} & =s c\left(\boldsymbol{J}^{T} \boldsymbol{J}\right)+\boldsymbol{\Lambda}_{0} \\
m^{(t+1)} & =\boldsymbol{\Lambda}^{-1}\left(s c \boldsymbol{J}^{T}\left(\boldsymbol{k}+\boldsymbol{J} m^{(t)}\right)+\boldsymbol{\Lambda}_{0} m_{0}\right) \\
s & =\left(\frac{1}{s_{0}}+\frac{1}{2} \boldsymbol{k}^{T} \boldsymbol{k}+\frac{1}{2} \operatorname{trace}\left(\boldsymbol{\Lambda}^{-1} \boldsymbol{J}^{T} \boldsymbol{J}\right)\right)^{-1}
\end{aligned}
$$

(iteration indices $t$ are only shown for $m$ as the other parameters are not dependent on their previous values). The EM-algorithm was initiated using the parameter values of the prior: $m^{(0)}=\hat{q}_{\mathrm{SVD}}, \boldsymbol{\Lambda}^{(0)}=\boldsymbol{\Lambda}_{0}, s^{(0)}=s_{0}$. The convergence of the variational posterior to the true posterior is monitored via the free energy $F(=$ lower bound of the model evidence): ${ }^{4}$

$$
\begin{aligned}
F= & -\frac{s c}{s_{0}}+\left(\frac{3 n}{2}+c_{0}-1\right)[\log (s)+\psi(c)] \\
& -\frac{1}{2}\left[\left(m-m_{0}\right)^{T} \boldsymbol{\Lambda}_{0}\left(m-m_{0}\right)+\operatorname{trace}\left(\boldsymbol{\Lambda}^{-1} \boldsymbol{\Lambda}_{0}\right)\right] \\
& -\frac{1}{2}\left[\boldsymbol{k}^{T} \boldsymbol{k}+\operatorname{trace}\left(\boldsymbol{\Lambda}^{-1} \boldsymbol{J}^{T} \boldsymbol{J}\right)\right]-s \log (c)-\log (\Gamma(c)) \\
& -c+\left(\frac{3 n}{2}+c-1\right)[\log (s)+\psi(c)]+\frac{1}{2} \log (\operatorname{det}(\boldsymbol{\Lambda}))+\text { constant }
\end{aligned}
$$

Maximizing the free energy is equivalent to minimizing the Kullback-Leibler distance between the true and approximated posteriors (Chappell et al., 2009). In the simulations, we considered the algorithm to be converged when the free energy changed less than $0.001 \%$ between successive iterations.

Chappell et al. (2009) show that this Variational solution gives the exact same point estimates as NLLS in the absence of prior information. In the simulations, we used a weakly informative prior on the rotation (normal prior centered at the SVD estimate and using a very small but non-zero precision) and a shrinkage prior on the precision, yielding slightly better results than NLLS at the lowest noise levels.

\section{Overview}

An overview of the different IK methods can be found in Table 1. Note that within the general classification of the methods, other algorithms are available than the ones used here.

Table 1: Overview of IK methods' classification and algorihtms.

\begin{tabular}{llll}
\hline abbreviation & reference & general classification & algorithm \\
\hline SVD & Soderkvist and Wedin (1993) & linear least sqauares & singular value decomposition \\
lsQUAT & Horn (1987) & linear least squares & eigenvalue decomposition \\
NLLS & Chapell et al. (2009) & non-linear least squares & Gauss-Newton iteration \\
VBIK & Chapell et al. (2009) & approximate Bayesian & Variational inference (Expectation Maximization) \\
BIK & Pataky et al. (2019) & full Bayesian & adaptive Metropolis-Hastings MCMC \\
\hline
\end{tabular}

\section{References}

See main paper

\footnotetext{
${ }^{4}$ In the free energy equation $\Gamma($.$) and \psi($.$) are the gamma and di-gamma functions respectively$
} 


\section{A comparison of three-dimensional rotation formalisms for least-squares and Bayesian inverse kinematics Appendix $\mathrm{C}$}

Ben Serrien, Klevis Aliaj, Todd Pataky
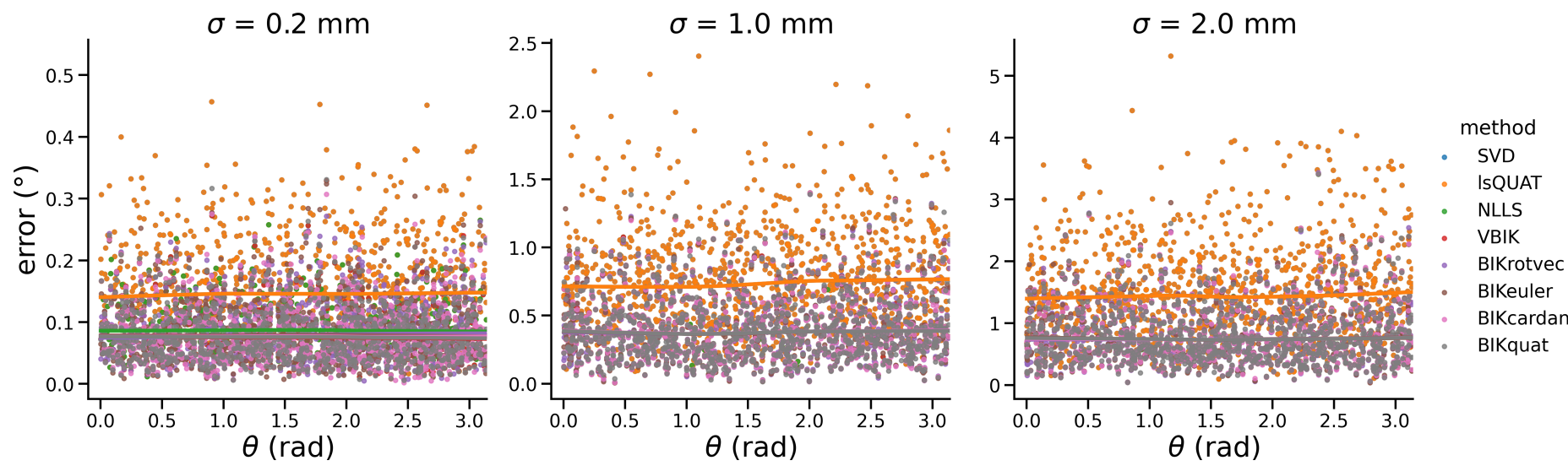

Figure 1: Scatter plots of inverse kinematics estimation errors versus rotation magnitude ( $\theta)$. Note the different $y$-axis scales corresponding to different noise levels $(\sigma)$. The lines are locally weighted scatter plot smoothers (LOESS) representing local averages.
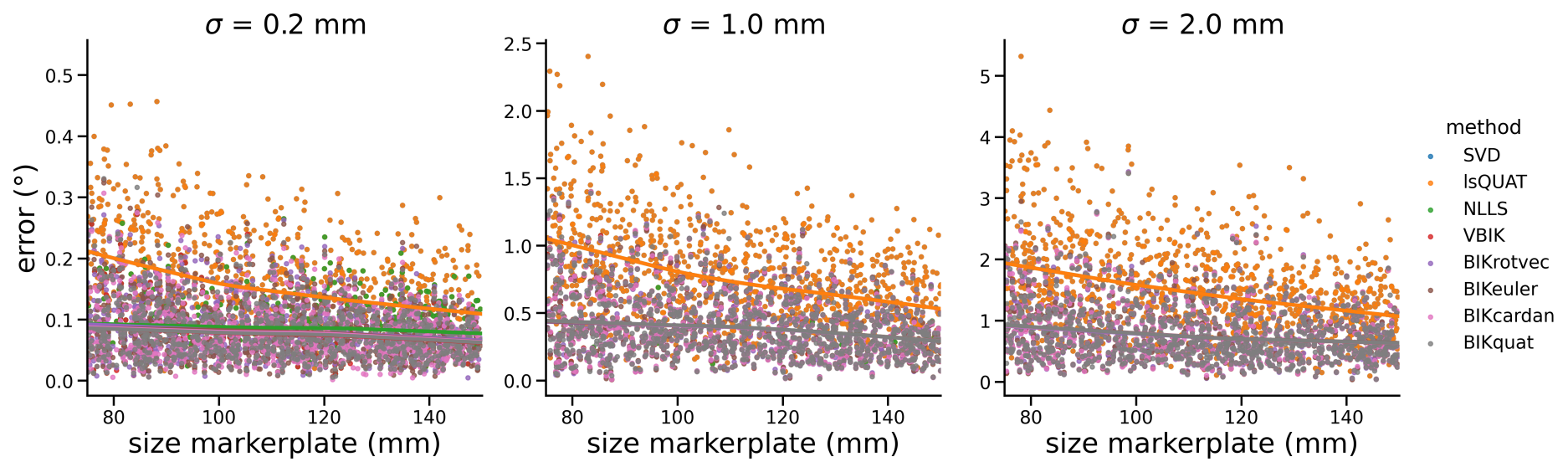

Figure 2: Scatter plots of inverse kinematics estimation errors versus size of the marker plate (size $=x$ indicates a square marker plate of $\left.x^{2} \mathrm{~mm}^{2}\right)$. Note the different y-axis scales corresponding to different noise levels $(\sigma)$. The lines are locally weighted scatter plot smoothers (LOESS) representing local averages.
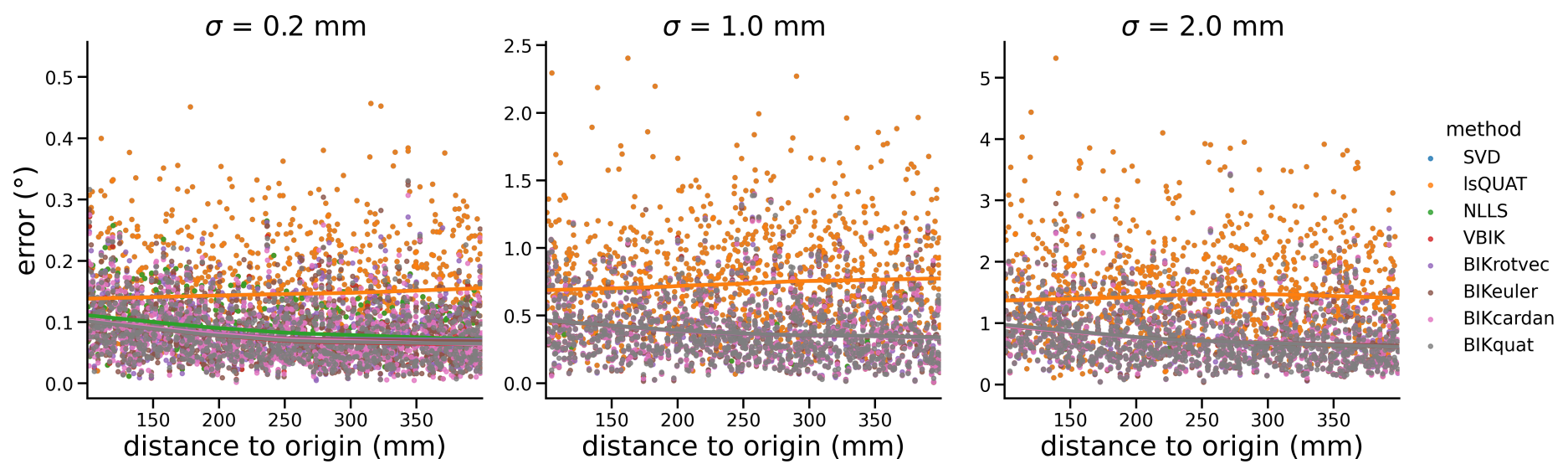

Figure 3: Scatter plots of inverse kinematics estimation errors versus distance of the marker plate centroid to the origin. Note the different $y$-axis scales corresponding to different noise levels $(\sigma)$. The lines are locally weighted scatter plot smoothers (LOESS) representing local averages. 
Table 1: Descriptive statistics of the IK errors (degrees)

\begin{tabular}{llccccccc}
\hline $\begin{array}{l}\text { noise } \\
\sigma(\mathrm{mm})\end{array}$ & method & mean & SD & min & Q1 & Q2 & Q3 & $\max$ \\
\hline 0.2 & BIKcardan & 0.083 & 0.045 & 0.002 & 0.050 & 0.074 & 0.105 & 0.307 \\
& BIKeuler & 0.085 & 0.046 & 0.008 & 0.051 & 0.077 & 0.108 & 0.324 \\
& BIKquat & 0.081 & 0.044 & 0.006 & 0.048 & 0.073 & 0.104 & 0.331 \\
& BIKrotvec & 0.086 & 0.045 & 0.005 & 0.054 & 0.077 & 0.107 & 0.276 \\
& NLLS & 0.091 & 0.045 & 0.007 & 0.057 & 0.085 & 0.117 & 0.329 \\
& SVD & 0.154 & 0.074 & 0.012 & 0.101 & 0.139 & 0.195 & 0.457 \\
& VBIK & 0.081 & 0.044 & 0.007 & 0.048 & 0.072 & 0.103 & 0.329 \\
& lsQUAT & 0.154 & 0.074 & 0.012 & 0.101 & 0.139 & 0.195 & 0.457 \\
1.0 & BIKcardan & 0.409 & 0.229 & 0.010 & 0.245 & 0.364 & 0.526 & 1.400 \\
& BIKeuler & 0.410 & 0.231 & 0.007 & 0.246 & 0.366 & 0.524 & 1.402 \\
& BIKquat & 0.413 & 0.234 & 0.015 & 0.248 & 0.367 & 0.522 & 1.389 \\
& BIKrotvec & 0.409 & 0.230 & 0.011 & 0.243 & 0.364 & 0.525 & 1.390 \\
& NLLS & 0.409 & 0.228 & 0.007 & 0.244 & 0.364 & 0.524 & 1.398 \\
& SVD & 0.772 & 0.370 & 0.067 & 0.492 & 0.731 & 0.986 & 2.404 \\
& VBIK & 0.409 & 0.229 & 0.007 & 0.244 & 0.365 & 0.525 & 1.398 \\
& lsQUAT & 0.772 & 0.370 & 0.067 & 0.492 & 0.731 & 0.986 & 2.404 \\
2.0 & BIKcardan & 0.809 & 0.457 & 0.042 & 0.479 & 0.700 & 1.057 & 3.414 \\
& BIKeuler & 0.813 & 0.456 & 0.037 & 0.487 & 0.701 & 1.061 & 3.415 \\
& BIKquat & 0.813 & 0.460 & 0.040 & 0.479 & 0.696 & 1.060 & 3.412 \\
& BIKrotvec & 0.807 & 0.455 & 0.047 & 0.478 & 0.689 & 1.055 & 3.432 \\
& NLLS & 0.807 & 0.454 & 0.047 & 0.480 & 0.699 & 1.054 & 3.410 \\
& SVD & 1.533 & 0.747 & 0.099 & 1.010 & 1.437 & 1.920 & 5.319 \\
& VBIK & 0.807 & 0.454 & 0.046 & 0.480 & 0.699 & 1.056 & 3.410 \\
& lsQUAT & 1.533 & 0.747 & 0.099 & 1.010 & 1.437 & 1.920 & 5.319 \\
\hline
\end{tabular}

